\title{
Population Density and Facility Type Influence Management of Prostate Cancer
}

\author{
Anjali Lankford ${ }^{1}$, Christian S DiBiase ${ }^{2}$, Sudesh Srivastav ${ }^{1}$, Nickolas Scherzer ${ }^{1}$, Jonathan Silberstein ${ }^{1}$ and Steven \\ $\mathrm{J} \mathrm{DiBiase}^{3 *}$
}

${ }^{1}$ Tulane University School of Medicine, New Orleans, LA, USA

${ }^{2}$ Boston College, Chestnut Hill, MA, USA

${ }^{3}$ Weill Cornell Medical College, New York, USA

Submission: January 07, 2021; Published: January 19, 2021

*Corresponding author: Steven J DiBiase, MD, Department of Radiation Oncology, Weill Cornell Medical College,1300 York Avenue, New York, NY, 10065, USA

\section{Abstract}

Objective: To investigate the role of population density and facility types on first course treatment for men with prostate cancer in the United States.

Methods: Using the National Cancer Database, we identified men diagnosed with prostate cancer from 2004-2015. Counties were sorted by population size and urbanization: metropolitan (metro), urban, and rural. Facilities were grouped by structural characteristics: community cancer centers and academic/research programs. Treatment was recorded as radiotherapy, radical prostatectomy or active surveillance. Two Chi-Square tests were used with significance level of $1 \%$ to assess the associations between facility type and urban/rural counties and frequency of treatment modalities. All analyses and summaries were performed using SAS software.

Results: We identified 1,277,411 men with prostate cancer. Modality usage varied $(\mathrm{p}=<0.0001)$ based on population density. The highest frequency of radiation was in urban counties $(48.40 \%)$. Surgery utilization was highest in rural counties (58.60\%). The highest rate of active surveillance was in metropolitan counties (2.88\%). Utilization also varied based on facility type, indicating academic programs employed higher frequencies of radiation $(43.20 \%)$ and active surveillance $(3.97 \%)$ while community cancer centers employed a higher frequency of prostatectomies $(63.63 \%)$.

Conclusion: Population density and facility type differences exist in the United States regarding management for prostate cancer, with a higher frequency of active surveillance in metropolitan counties, radiation in urban counties, and surgery in rural areas. Lastly, community cancer centers had higher rates of radiation while academic centers more frequently employed prostatectomies and active surveillance.

Keywords: Prostate cancer; Population density; Radiotherapy; Watchful waiting; Academic medical centers

\section{Introduction}

Prostate cancer $(\mathrm{PCa})$ is the most commonly diagnosed cancer and second leading cause of cancer deaths for men in the United States. The United States experienced 233,000 newly diagnosed cases and more than 29,000 deaths in 2014 [1]. Prostate specific antigen (PSA) screenings have historically been used as means for PCa early detection. However, in 2012, the United States Preventative Services Task Force (USPSTF) updated the PCa screening guidelines to discourage physicians from PSA screenings on men of all ages. USPSTF has since updated their guidelines again to indicate that the choice to undergo PSA screenings should be up to the patient for those ages 55-69. Patients are encouraged to have a discussion with their physician about the risks and benefits. Since the 2012 update to guidelines, the U.S. has seen a decline in prostate biopsies and PCa incidence; however, the malignancies that are diagnosed are higher grade and stage upon detection [2]. Though the prevalence of localized PCa among U.S. men is declining, it still causes significant morbidity and mortality. Men with PCa have several treatment options with which to manage their cancer. The most common options are radiation therapy (RT), surgery (radical prostatectomy [RP]), 


\section{Cancer Therapy \& Oncology International Journal}

or active surveillance (AS). Many factors may go into choosing a management option (e.g. availability, accessibility, preference) but the standard of care is not as well delineated as it is for other malignancies. Thus, we aimed to study the factors that may impact this decision for patients and physicians. More specifically, we sought to investigate the role of population density and cancer center facility type on utilization of RP, RT, and AS treatment options to manage localized PCa in the United States.

\section{Methods and Materials}

The National Cancer Database (NCDB) is an online oncology database sourced from facility registry data that are collected in more than 1500 Commission on Cancer (CoC)-accredited facilities. This large database is a joint effort between the Commission on Cancer of the American College of Surgeons and the American Cancer Society. Reviews of the NCDB reflect its aim to be a useful representative of cancer care across a broad range of hospital cancer registries in the United States $[3,4]$. NCDB data are collected using a standardized electronic format that has been described elsewhere. ${ }^{4}$ Hospital participation is voluntary; data collection and quality assurance is overseen by hospital cancer committees. Though the $\mathrm{CoC}$ of the American College of Surgeons and the American Cancer Society are responsible for the collection and distribution of the data, neither organization is responsible for the present analysis or conclusions.

Using the NCDB, we isolated men with a diagnosis of localized prostate cancer in the United States between 2004 and 2015. Localized PCa was defined as non-metastatic prostate cancer that consisted of American Joint Commission on Cancer (AJCC) stages I through III [5]. Any incomplete data points were excluded from the study.

The first course of treatment was recorded as either any type of radiation therapy (RT), radical prostatectomy (RP) or active surveillance (AS). The RT group contained but was not limited to patients receiving external beam radiation (EBRT) or brachytherapy (BT), or RT plus androgen deprivation therapy (ADT) since RT was the main treatment modality. ADT included both medical and surgical therapy (orchiectomy). The RP group included patients receiving either RP or RP combined with RT or $\mathrm{ADT}$, since surgery was the primary treatment recommendation. $\mathrm{RP}$ was defined as total prostatectomy, which includes the removal of prostate, ejaculatory duct and seminal vesicles with or without lymph node dissection; however localized treatments for PCa are gaining in popularity. AS was defined as when a patient undergoes further biopsies and imaging, postponing or ultimately avoiding primary treatment; this differs from no treatment due to the watchful nature. Patients who received no first course treatment or ADT only were excluded from analysis. Patients who received another treatment not previously mentioned were also excluded. This exclusion criteria included those who received subtotal prostatectomy or any surgery aside from radical prostatectomy such as cystoprostatectomy. Patients who received chemotherapy, biologic response modifiers or experimental treatment were also excluded. Transurethral resection of prostate was recorded as no treatment as it was not classified as cancer directed.

To assess facility type, facilities were classified by the Commission on Cancer ( $\mathrm{CoC}$ ) Accreditation Program based on structural characteristics. This classification system included four types of facilities: Integrated Network Cancer Programs (INCP), Community Cancer Programs (CCP), Comprehensive Community Cancer Programs (CCCP) and Academic/Research Programs (ARP). INCP are defined as a collection of facilities owned by an organization with one facility being a CoC-accredited hospital and all facilities offering comprehensive services. A CCP is a facility that caters to between 100-500 newly diagnosed cancer cases per year and provides a variety of treatment services. A CCCP is a larger version of a CCP and oversees more than 500 new cancer diagnoses per year. Lastly, an ARP is a facility that sees over 500 new cancer cases per year and offers postgraduate medical education in at least four specialties, including internal medicine and general surgery. The ARP category also considered National Cancer Institute (NCI)-designated Comprehensive Cancer Centers, which are facilities with NCI peer-reviewed grants and no minimum caseload requirement. These NCI-designated centers only make up a small portion (3\%) of all CoC-accredited facilities [6]. Due to structural similarities, Community Cancer Programs, Comprehensive Community Cancer Programs, and Integrated Network Cancer Programs were combined to form Community Cancer Centers (CCC) for this review and were compared to a second group, Academic/Research Programs (ARP).

To assess population density as an influencing factor in PCa management, the U.S. was divided into three groups based on codification of population size and urbanization by the United States Department of Agriculture (USDA) Economic Research Service and Office of Management and Budget (OMB). The patient's address was estimated by using state and county Federal Information Processing Standard (FIPS) codes in patient records at the time of diagnosis. Depending on the county in which the patient resided, the patient's location was identified as one of three categories: metropolitan, urban, and rural. Metropolitan (metro) areas were subdivided into three groups based on population size while nonmetropolitan areas were subdivided into nine groups of urban or rural based on degree of urbanization and adjacency to a metro area. For simplicity, our study-based population density on the three major categories of metropolitan, urban and rural. According to the USDA Rural-Urban Continuum used in the NCDB, metro counties were defined as areas with urbanized sections or a $25 \%$ commuter rate regardless of population size. Urban counties were areas not a part of a larger labor market and had a population of 2,500 - 49,999 residents. Rural counties included either towns with less than 2,500 people or open countryside [7].

All data for study specific variables were summarized using descriptive statistics. Frequency tables were made for nominal and ordinal data. A $\chi^{2}$ test assessed the association between both 


\section{Cancer Therapy \& Oncology International Journal}

population density and facility type and the frequency of RT, RP, and AS. A 2-sided 5\% significance level was used throughout the analyses. All analyses, summaries, and listings were performed using SAS software (version 9 or higher in a Windows environment).

\section{Results}

This study identified 1,277,411 men with localized prostate cancer treated in the U.S., of whom 57.32\% underwent RP, 40.11\% underwent RT and $2.05 \%$ chose AS. Using AJCC staging, $12.08 \%$ of patients were in stage I, $76.83 \%$ were in stage II and $11.09 \%$ were in stage III. Table 1 illustrates the number of patients evaluated based on population density, facility type, and elected first course treatment. When comparing the utilization of different management options based on population density, significant differences were observed (Figure 1). A $\chi^{2}$ analysis of the data yielded a P-value of $<.0001$. In urban counties, RT was chosen in $48.40 \%$ of patient cases, while it was used significant less in metro $(42.41 \%)$ and rural $(38.01 \%)$ counties $(p=<0.0001)$. RP utilization had an opposite trend, with RP being more frequently chosen by patients from rural counties (58.60\%) compared to those from urban $(48.40 \%)$ and metro $(54.71 \%)$ counties $(\mathrm{p}=<0.0001)$. AS usage patterns also varied between metro (2.88\%), urban (2.07\%), and rural areas (2.49\%). These AS data were also statistically significant $(\mathrm{p}=<0.0001)$.

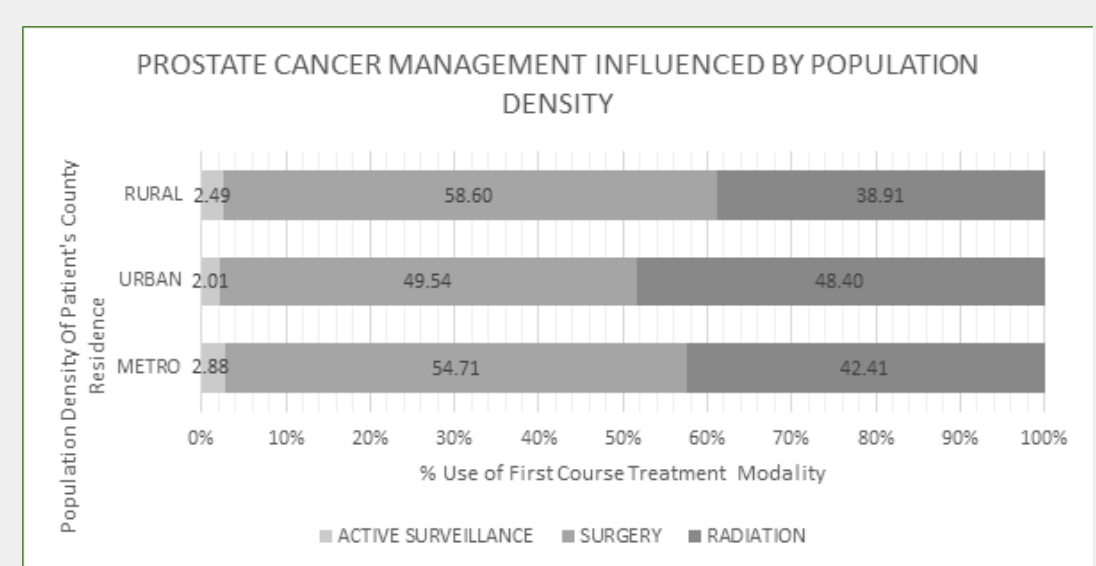

Figure 1: Population density of patient's home address influences use of radiation, surgery or active surveillance for prostate cancer treatment.

As seen in Figure 2m utilization of AS, RP, and RT varied based on facility type; $\mathrm{a} \chi^{2}$ analysis of the data yielded a P-value of $<.0001$. Data indicated a higher frequency of RT $(43.20 \%)$ at CCC than at ARP $(32.40 \%)(p=<0.0001)$. There was a significant difference $(\mathrm{p}=<0.0001)$ between the frequency of RP at ARP $(63.63 \%)$ compared to CCC (55.00\%). Lastly, there was a significant difference $(\mathrm{p}=<0.0001)$ in the frequency of AS occurring at ARP $(3.97 \%)$ compared to CCC $(1.80 \%)$.

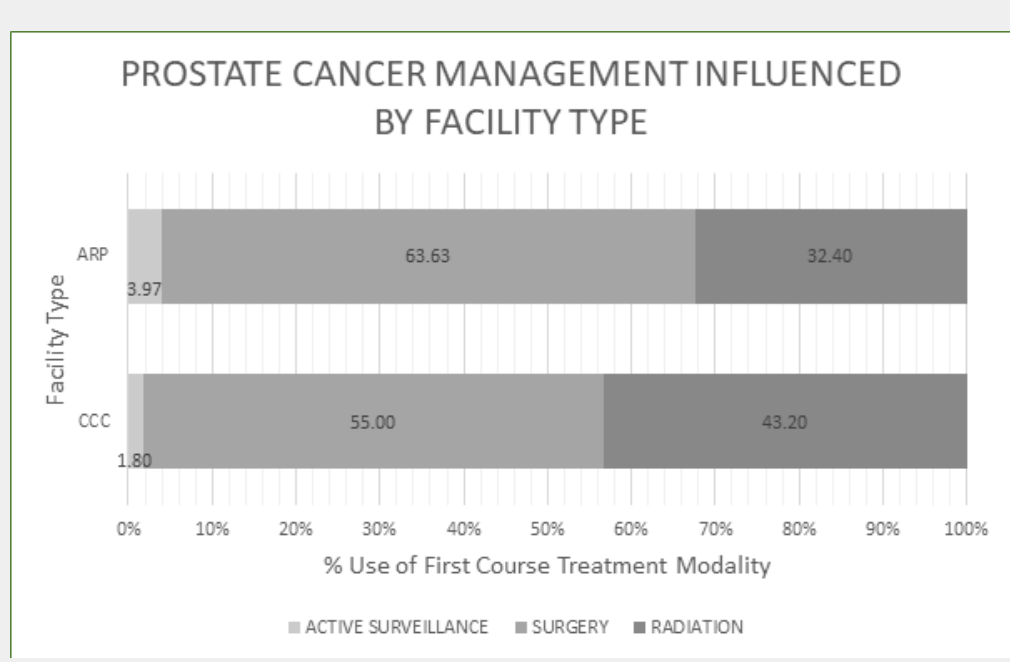

Figure 2: Facility type differences in radiation, surgery, or active surveillance for localized prostate cancer treatment. 


\section{Cancer Therapy \& Oncology International Journal}

Table 1: Number of Prostate Cancer Patients Receiving Each Treatment Modality Based on Population Density in the United States, $2004-2015$. Percentages of values are shown in parentheses.

\begin{tabular}{|c|c|c|c|c|}
\hline & Metropolitan Counties & Urban Counties & Rural Counties & TOTAL \\
\hline AS $^{\mathbf{1}}(\%)$ & $26657(2.88)$ & $4671(2.01)$ & $700(2.49)$ & $32028(2.71)$ \\
\hline Surgery (\%) & $505740(54.71)$ & $112022(49.54)$ & $16448(58.60)$ & $634210(53.81)$ \\
\hline Radiation (\%) & $392009(42.41)$ & $109445(48.40)$ & $10920(38.91)$ & $512374(43.47)$ \\
\hline TOTAL & $924406(78.43)$ & $226138(19.19)$ & $28068(2.38)$ & 1178612 \\
\hline
\end{tabular}

${ }^{1}$ Active Surveillance

\section{Discussion}

The definitive management of localized prostate cancer is controversial due to a lack of randomized studies showing significant differences in outcomes. In the well-known Protect study, a 10-year follow-up study published in 2016, rates of prostate-cancer specific deaths were determined for men with localized PCa who received either RP, RT, or AS. This study found no significant differences in 10-year outcomes between the three treatment options. However, RP and RT were found to have lower incidence of disease progression and metastases than AS [8]. Thus, since patient survival appears to be similar, many other factors are likely involved in choosing a treatment for localized PCa. In this paper, when examining the influence of population density and cancer center type on the type of treatment used in over 1.2 million men with PCa, we discovered significant differences.

Our study identified significant differences in the utilization of RP, RT, and AS in patients based on population differences. In particular, patients from urban areas received the highest frequency of RT and patients from rural areas received the highest frequency of RP. There was also a statistical difference in AS rates based on population density with patients living in metro counties receiving the highest rates of AS when compared to urban and rural counties.

As for facility type, our study also yielded significant differences between treatment modalities. Patients at CCCs received more RT and less RP whereas patients at ARPs were more likely to get RP and less RT. Our findings on facility type differences were consistent with another NCDB retrospective study done from 2004-2013 by Löppenberg et al. which revealed community care centers used the highest rates of RT and lowest rates of RP. The lowest rates of brachytherapy, a form of RT, was noted at academic facilities [9]. Our findings are consistent with those previously reported in a study done by Camarata et al that showed an increase usage of EBRT at independent cancer centers. Similar to CCC in our study, when comparing independent cancer centers to larger multispecialty centers, like most ARP, a difference was noted.

Similar to definitive treatment options of surgery and radiation therapy, AS utilization was also affected by cancer center type in this report. At ARPs, AS was chosen more frequently at
ARPs, which is consistent with previously reported data $[10,11]$. Aizer et al reported that patients were more likely to choose AS if they obtained a consultation at a multidisciplinary care clinic than with individual practitioners [11]. The choice of an AS recommendation is likely a complex decision and may be influenced by many variables included center type.

The influence of other sociodemographic differences in PCa treatment modality have been noted by previous investigators to influence treatment recommendation. In the previous noted study by Aizer et al, factors such as age, marital status, and physician experience appeared to influence treatment decision [11]. Interestingly, Woodhouse et al noted that the type of specific radiation modality recommended could be influenced by non-cancerous/clinical factors. An urban academic facility showed significant differences in the use of proton beam therapy as opposed to photon radiation therapy intensity modulated radiation therapy (IMRT). Further, IMRT was more frequently used for patients who were older, black, and lived closer to the facility [12].proton therapy (PT Camarata et al also reported the influence of age and race on the recommendation to undergo EBRT [13].

Another variable that may play a significant role in treatment choice for patients considering RT in rural versus metropolitan or urban areas is the travel distance and associated convenience of undergoing treatment. Most men with localized PCa who receive EBRT will be under treatment for typically 5-8 weeks. Consequently, the length of treatment for EBRT may sway rural patients with a longer commute to seek a more convenient, expeditious treatment, such as RP. Other investigators have confirmed a negative influence of treatment distance on RT utilization [14]. However, a newer form of RT called stereotactic body radiation therapy (SBRT) which utilizes a hypofractionated radiation approach of high radiation doses delivered over one week may challenge this point, and current studies show comparable, if not better results to conventional RT approaches $[15,16]$. The shorter treatment time may make RT much more accessible to patients, particularly those in rural areas who may travel farther to receive treatment. So as usage of SBRT increases, perhaps it will impact who receives RT versus other forms of treatment in the future. 


\section{Cancer Therapy \& Oncology International Journal}

Both RP and RT are associated with treatment-related side effects which must be considered before a patient chooses a particular treatment. In the report by Chen et al., RT patients reported a higher incidence of sexual dysfunction and acute worsening of urinary obstruction following 3 months post treatment compared to patients pursuing AS. RP patients suffered from increased average rates of sexual dysfunction and urinary incontinence compared to AS patients for the 3 months following treatment [19]. These side effects can greatly reduce a patient's quality of life and may factor into the treatment decision.

There are likely numerous reasons the explain the differences in localized PCa treatment across facility types and population densities. Cultural differences and patient preference likely play a role but are difficult to isolate and quantify. Another potential factor may include physician preference. In a report by Bekleman et al., financial compensation may influence physician treatment recommendations. This study reported that as the Medicare reimbursement for hormone therapy decreased, so did the rate of usage. Simultaneously, Medicare reimbursement for EBRT was much higher than RP. So as urology practices converted to integrated prostate cancer centers that could now offer RT, rates of RT usage increased [20].

Limitations of our study include its retrospective nature. In addition, our study includes Integrated Network Cancer Centers as a part of the CCC category when considering facility type. However, Integrated Network Cancer Programs can be composed of several different types of facilities. The most up to date data that we have access to is from 2015. Practice patterns have changed since 2015, such as the increasing popularity of active surveillance, but we hope that this study can identify trends that are still applicable today [21]. Lastly, if a patient undergoing AS undergoes treatment later, the NCDB categorizes that patient as undergoing subsequent treatment instead of AS. Therefore, we were unable to account for men who received AS before treatment, which means our results could underestimate the AS figure [22].

Differences in facility type and the population density of a patient's home county influence definitive treatment for localized prostate cancer in the United States. The causes behind these differences are likely complex. Though our study does not aim to provide specific reasons for the observed differences in treatment options, we hope that these data will be meaningful and allow patients and physicians to better understand their choices and how they may relate to external factors. Additional research on treatment decision-making, patient attitudes toward different modalities and patient-care differences between facility is necessary to improve our understanding of how patients choose a first-course treatment for localized prostate cancer.

Data from the National Cancer Database was incorporated into a visual representation of the percentages of localized prostate cancer patients who underwent Active Surveillance, Surgery (radical prostatectomy), or Radiation in three population density categories (metro, urban, rural) between the years of 2004-2015 (Table 2). Metropolitan couties are the most population dense, followed by urban counties, and lastly, rural counties are the least population dense (Figure 1).

Table 2: Number of Prostate Cancer Patients Receiving Each Treatment Modality Based on Facility Type in the United States, 2004-2015. Percentages of values are shown in parentheses.

\begin{tabular}{|c|c|c|c|}
\hline & Community Cancer Center & Academic/Research Program & TOTAL (\%) \\
\hline AS $^{2}$ (\%) & $14142(1.80)$ & $18669(3.97)$ & $732811(2.61)$ \\
\hline Surgery (\%) & $433092(55.00)$ & $299134(63.63)$ & $492558(39.17)$ \\
\hline Radiation (\%) & $340215(43.20)$ & $152343(32.40)$ & 1257595 \\
\hline TOTAL & $787449(62.62)$ & $470146(37.38)$ & \\
\hline
\end{tabular}

${ }^{2}$ Active Surveillance

Results: There was statistically significant differences $(\mathrm{p}=<0.0001)$ in the utilization each modalities depending on the population density of the patient's home address. Radiation was used most frequently in urban areas (48.40\%). Surgery utilization was highest in rural areas (58.60\%). Active surveillance usage was highest in metro counties (2.88\%).

Data from the National Cancer Database was incorporated into a visual representation to compare the percentages of localized prostate cancer patients who underwent Active Surveillance, Surgery (radical prostatectomy), or Radiation in Community Cancer Centers (CCC) versus Academic/Research Programs (ARP) between the years of 2004-2015 (Figure 2).
Results: There were statistically significant differences in modality usage between CCC and ARP $(p=<0.0001)$. There was a higher frequency of Active Surveillance occurring at ARP (3.97\%) compared to CCC $(1.80 \%)$. Radiation was used more at CCC (43.20\%) than ARP (32.40\%). Surgery was used more at ARP (63.63\%) compared to CCC $(55.00 \%)$.

\section{References}

1. Scher HI, Solo K, Valant J, Todd MB, Mehra M (2015) Prevalence of Prostate Cancer Clinical States and Mortality in the United States: Estimates Using a Dynamic Progression Model. PLoS One 10(10): e0139440. 


\section{Cancer Therapy \& Oncology International Journal}

2. Fleshner K, Carlsson SV, RM (2017) The effect of the USPSTF PSA screening recommendation on prostate cancer incidence patterns in the USA. Nat Rev Urol 2 14(1): 26-37.

3. Stewart AK, Bland KI, McGinnis LS, Morrow M, Eyre HJ (2000) Clinical highlights from the National Cancer Data Base, 2000. CA Cancer J Clin 50(3): 171-183.

4. Menck HR, Garfinkel L, Dodd GD (1991) Preliminary Report of the National Cancer Data Base 41(1): 7-18.

5. Stephens NJ, Bharwani N, Heenan SD (2008) Prostate cancer staging. Imaging 20(2):112-121.

6. American College of Surgeons, American Cancer Society. National Cancer Database. Natl Cancer Database 2017: 500.

7. United States Department of Agriculture. Economic Research Services. 2013 Rural-Urban Continuum Codes.

8. https://www.ers.usda.gov/data-products/rural-urban-continuumcodes/documentation/

9. Hamdy FC, Donovan JL, Lane JA, Malcolm Mason, Chris Metcalfe, et al. (2016) Ten-year Outcomes after Monitoring, Surgery, or Radiotherapy for Localized Prostate Cancer. N Engl J Med 375(15): 1415-1424.

10. Löppenberg B, Sood A, Dalela D, Patrick Karabon, Jesse D Sammon, et al. (2017) Variation in Locoregional Prostate Cancer Care and Treatment Trends at Commission on Cancer Designated Facilities: A National Cancer Data Base Analysis 2004 to 2013. Clin Genitourin Cancer 15(6): e955-e968.

11. Lester-Coll NH, Park HS, Rutter CE, Christopher D Corso, Brandon R Mancini, et al. (2016) The Association Between Evaluation at Academic Centers and the Likelihood of Expectant Management in Low-risk Prostate Cancer. Urology 96: 128-135.

12. Aizer AA, Paly JJ, Zietman AL, Paul L Nguyen, Clair J Beard, et al. (2012) Multidisciplinary care and pursuit of active surveillance in low-risk prostate cancer. J Clin Oncol 30(25): 3071-3076.

13. Woodhouse KD, Hwang WT, Vapiwala N, Akansha Jain, Xingmei Wang, et al. (2017) Sociodemographic disparities in the utilization of proton therapy for prostate cancer at an urban academic center. Adv Radiat Oncol 2(2):132-139.
14. Camarata AS, Nickleach DC, Jani AB, Rossi PJ (2015) Locoregional Prostate Cancer Treatment Pattern Variation in Independent Cancer Centers: Policy Effect, Patient Preference, or Physician Incentive? Heal Serv Insights 8: 1-8.

15. Muralidhar V, Rose BS, Chen YW, Nezolosky MD, Nguyen PL (2016) Association Between Travel Distance and Choice of Treatment for Prostate Cancer: Does Geography Reduce Patient Choice? Int J Radiat Oncol Biol Phys 96(2): 313-317.

16. Anwar M, Weinberg V, Chang AJ, Hsu IC, Roach M, et al. (2014) Hypofractionated SBRT versus conventionally fractionated EBRT for p... - PubMed - NCBI. Radiat Oncol 9(1): 42.

17. Catton CN, Lukka H, Julian JA, Jarad M Martin, Stéphane Supiot, et al. (2016) A randomized trial of a shorter radiation fractionation schedule for the treatment of localized prostate cancer. J Clin Oncol 34(15_suppl): 5003-5003.

18. Hellekson C, Larson G, Poswilko S, Beal J, Sahmoun A, Russo JK (2016) On the Association between Travel Distance and Treatment Choice for Low-Risk Prostate Cancer: Results from a Rural State. J Cancer Ther 07(09): 656-664.

19. Baldwin LM, Andrilla CHA, Porter MP, Rosenblatt RA, Patel S, et al (2013) Treatment of early-stage prostate cancer among rural and urban patients. Cancer 119(16): 3067-3075.

20. Chen RC, Basak R, Meyer AM, Tzy-Mey Kuo, William R Carpenter, et al (2017) Association between choice of radical prostatectomy, external beam radiotherapy, brachytherapy, or active surveillance and patientreported quality of life among men with localized prostate cancer. JAMA - J Am Med Assoc 317(11): 1141-1150.

21. Bekelman JE, Suneja G, Guzzo T, Pollack CE, Armstrong K, et al. (2013) Effect of practice integration between urologists and radiation oncologists on prostate cancer treatment patterns. J Urol 190(1): 97101.

22. Kinsella N, Helleman J, Bruinsma S, Sigrid Carlsson, Declan Cahill, et al. (2018) Active surveillance for prostate cancer: A systematic review of contemporary worldwide practices. Transl Androl Urol 7(1): 83-97.

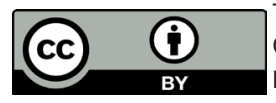

This work is licensed under Creative Commons Attribution 4.0 License DOI:10.19080/CTOIJ.2021.17.555973
Your next submission with Juniper Publishers will reach you the below assets

- Quality Editorial service

- Swift Peer Review

- Reprints availability

- E-prints Service

- Manuscript Podcast for convenient understanding

- Global attainment for your research

- Manuscript accessibility in different formats

(Pdf, E-pub, Full Text, Audio)

- Unceasing customer service

Track the below URL for one-step submission https://juniperpublishers.com/online-submission.php 